Review

\title{
Diagnosis of Composite Materials in Aircraft Applications-Brief Survey of Recent Literature
}

\author{
Muflih Alhammad 1, Luca Zanotti Fragonara ${ }^{2}$ and Nicolas P. Avdelidis 3 ,* \\ 1 Aerospace, Canfield University, Bedford MK43 0AL, UK; m.s.alhammad@cranfield.ac.uk \\ 2 Aerospace, Canfield University, Bedford MK43 OAL, UK \\ 3 Aerospace, Canfield University, Bedford MK43 0AL, UK \\ * Correspondence: np.avdel@cranfield.ac.uk.
}

\begin{abstract}
Diagnosis and prognosis of failures for aircrafts' integrity are some of the most important regular functionalities in complex and safety-critical aircraft structures. Further, development of failure diagnostic tools such as Non-Destructive Testing (NDT) techniques, in particular, for aircraft composite materials, has been seen as a subject of intensive research over the last decades. The need for diagnostic and prognostic tools for composite materials in aircraft applications rises and draws increasing attention. Yet, there is still an ongoing need for developing new failure diagnostic tools to respond to the rapid industrial development and complex machine design. Such tools will ease the early detection and isolation of developing defects and the prediction of damages propagation; thus allowing for early implementation of preventive maintenance and serve as a countermeasure to the potential of catastrophic failure. In this paper, following a short introductory summary and definitions, this paper provides a brief literature review of recent research on failure diagnosis of composite materials with an emphasis on the use of NDT techniques in aerospace industry. In addition to this, within a some of significant NDT application extents, prognosis of composites is also briefly discussed.
\end{abstract}

Keywords: Failure Diagnosis, Aircraft Applications, Composite Materials, Non-Destructive Testing (NDT) Techniques

\section{Introduction}

Composite materials have been utilized for various machining and industrial processes since last decades until now. Composite materials are so called because they are formed from two or more materials, one of which, the fibre, is scattered in a continuous matrix phase. The two materials work together so that they provide different material properties from the properties of the original elements on their own [1]. Though most airplanes today are made out of aluminum, composite materials such as those materials made from carbon fibres, glass fibres, and Kevlar, to name few, are widely used in the aircraft industry. For instance, glass fibres were first employed in aircraft, by Boeing, in the 1950s. Also, Boeing 787 Dreamliner was the first commercial airplane to be built from $50 \%$ composite materials, mostly carbon fibre composites [2-7]. Recent years have also seen a noticeable increase in the use of composite materials in aircraft industry compared with the other materials, shown in figure 1. The greatest advantage of composite materials usage is the lower-weight planes. For instance, they are stronger, yet lightweight than aluminium. This weight reduction will lead to more fuel-efficient planes that will require less fuel to propel themselves forward. High strength-to-weight ratio, also known as specific strength, is another advantage of the composites and as a result are incredibly str onger than the other metals used in aircraft manufacturing. Hence, they can resist compression loads and do not easily break under tension conditions.

Composite materials are also insusceptible to corrosion due to harsh chemical components, and their resistant to many highly reactive chemicals. Further, they can incredibly handle the exposure to severe weather conditions including a wide variation in temperature. This is very important in the aircraft industry as the most essential parts of any airplane made out of composite materials will 
neither grow nor shrink, or change as a result of varying environmental conditions. Design flexibility represents another big advantage of composite materials over the other metals. Composites can be formed and/or made into just about the required shape. For instance, a single oddly shaped piece of composite can replace many pieces made out of other materials. This helpful characteristic lead eventually not only to cut down the maintenance cost but also reduce the total cost over the lifetime of planes [2-7].

\section{Used Materials in Aircraft Industry 1985-2014}

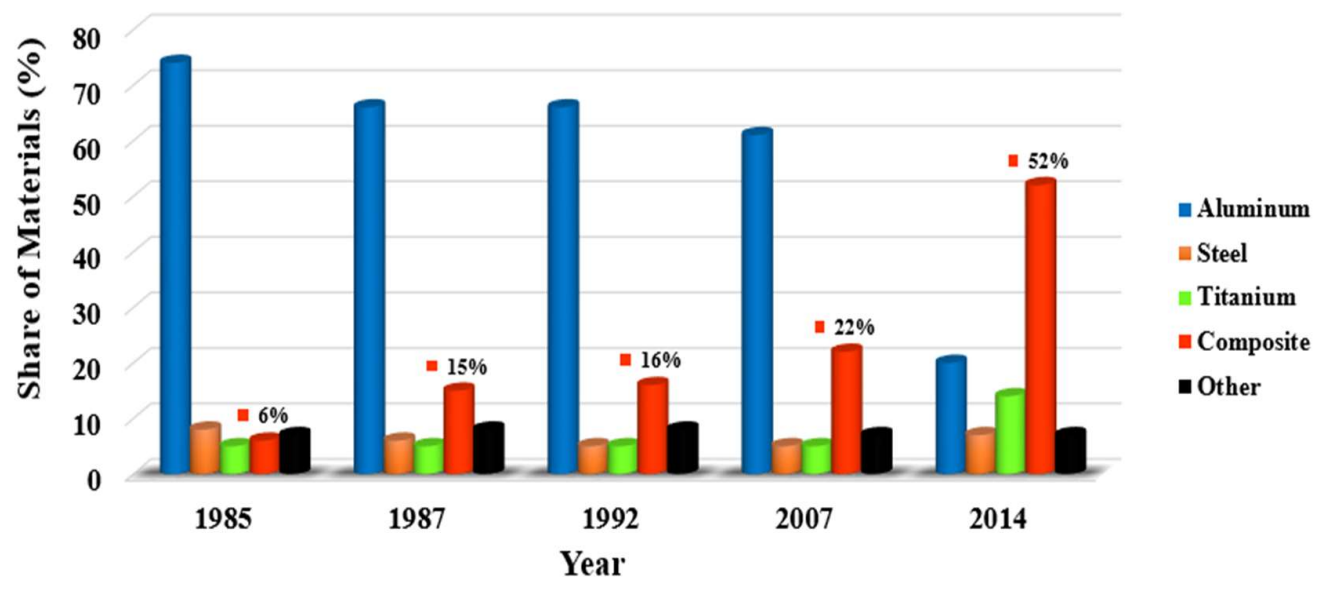

Figure 1. Used Materials in Aircraft Industry 1985-2014 [8].

Nevertheless, composite materials have revolutionized the aviation industry, they have, however, their own disadvantages compared with the alternative materials. Higher initial cost for aircraft and component manufacturers compared with the other metals is perhaps the biggest disadvantage of composite materials. This high initial cost is mainly attributed to the high price of fibres and the complicated manufacturing processes. Another disadvantage is that the prediction of failure time for the internal structure of any composite aircraft piece cannot easily be made. This, in turn, leads to very costly and difficult damage inspections. Also, during the inspections, delamination may occur, layers of composite separate, as a result of an impact to the composite piece. As consequence of this, water may penetrate to the delaminated composite piece, causing serious problems, in particular, when water freezes and thaws. Unlike, for instance, aluminium, most of standard composite materials used in aircraft industry do not conduct electricity, and therefore they cannot prevent, for example, lightning from being directed to the fuel tanks. However, it was reported that composites were integrated with wire mesh to fix this problem; this method was first used in 787 aircrafts. The existence of resin in the manufactured composite materials is reported as one of the causal factors behind the catastrophic failure. At temperatures below $65^{\circ} \mathrm{C}$, resin weakens the composites and yet it may also lead to aircraft failure when temperatures reach even as above as $150^{\circ} \mathrm{C}[2-7]$.

As it can be seen the use of composites in aircraft industry does present some engineering and maintenance challenges. This involves damage inspections and diagnosis that may lead to costly manufacturing and repairs. With more and more commerce and business being conducted every day in this field, analysis of data for detecting and repelling damage of composites is paramount to every aircraft company. To directly tackle and respond to the lack of the engineering improvement for the damage inspection techniques for composites in aircraft industry, off the shelf, tremendous work over a number of recent years was undertaken. Though several attempts were made to summarize this intensive number of published work, it is still unfortunately scattered here and there. There is a need to briefly lump/highlight this work in one single document so that provide the interested readers not only with an overall overview of the latest developed tools but also with an ease of making a survey whenever there is a need for it. This paper, as consequence of that, aims to: 
- Briefly provide a short preliminary overview and definitions and a summary of recent methodologies and tools in the area of failure diagnosis (FD).

- Bring together in one document most of the previous research attempts at the investigating of the most possibly viable Non-Destructive Testing (NDT) techniques, although there are a wide variety of NDT methods built upon different principles, for the inspection of composites in aircrafts' structures.

It should be telling that the failure diagnosis is a prior condition analysis to the prognosis, and hence, while it is very briefly highlighted in the paper, the emphasis of this review is mainly on the failure diagnosis of composites.

\section{2. Failure Inspections for Composite Materials}

Failure inspections or failure diagnosis can be defined as the process of analyzing physical parameters, associated with the structure, for the purpose of determining structure integrity [9]. Failure diagnosis involves the prediction of damage onset on a structure when subjected to loads and environmental conditions. Failure may consist permanent damages such as structural deformations (plastic deformation), or lack of functionality [9]. It is worth mentioning that crack/damage onset does not mean the final form or catastrophic failure of the structure. To keep, for instance, aircrafts function at optimal levels, condition monitoring of structural components (e.g. composites) is important because any wear, if is not caught in time, will often progress to more serious damage affecting the adjacent parts. In the following sections, a review of NDT and other failure diagnosis methods, which are possibly viable for composites in aircraft applications, to evaluate a specific type of damage identification is presented.

NDT refers to a method of detecting internal flaws in engineering materials without breaking them [10-11]. It can also be defined as the use of non-invasive technique to determine the integrity of a material, component or structure or quantitatively measure some characteristics of an object. It is very broad interdisciplinary field that plays a vital role in analysing the properties and parameters of any material [11]. Advantages of NDT methods include:

- Analysis of parts can be done without breaking them.

- Cost saving procedure.

- Improve the quality of production.

- $\quad$ Saves time in product evaluation.

- $\quad$ Evaluation can be done at manufacturing stage or in service stage.

- Portable mode of inspection.

- $\quad$ Surface defects and inside defects can be easily evaluated.

NDT techniques can be classified in many ways such as Conventional and Advanced methods, Passive and Active techniques or Contact and Non-Contact methods., etc., to name a few [12-15]. Conventional techniques are the NDT methods that have well-established and fully matured over the past decades. Yet, they are described as well-documented techniques in terms of codes, standards, and best practices. In contrast, advanced methods are very limited in use as they tend to be less understood due to either uncertain advantages or limitations, lack of laborious qualification criteria, or little to no industry codification. With the passive approach, NDT methods, e.g. acoustic emission, are used to investigate different materials/structures by reflecting their internal energy using proper physical fields, whilst in the case of the active approach an external stimulus source (forced physical field such as heat, force, cold air gun etc., to name few.) is used. Contact NDT type is commonly used in construction industry where, for instance, transducers are directly attached to the materials or structures under test; e.g. Ultrasonic testing. In the Non-contact methods, data are collected without the need for a direct accessibility to the surface of the structure being tested; e.g. Thermography test. Classification NDT techniques based on the category of contact or non-contact methods is presented in table 1.

Table 1 Contact and Non-Contact NDT Methods

\begin{tabular}{cc}
\hline Contact Methods & Non-Contact Methods \\
\hline Traditional Ultrasonic Testing & Through Transmission Ultrasonic
\end{tabular}




\begin{tabular}{cc} 
Acoustic Emission & Radiography Testing \\
Eddy Current Testing & Thermography \\
Magnetic Testing & Infrared Testing \\
Electromagnetic & Holography \\
Penetrant Testing & Shearography \\
Liquid Penetrant & Visual Inspection \\
\hline
\end{tabular}

Attempts were made to evaluate the integrity conditions of composites in aircraft applications using NDT techniques. In the literature, a number of case studies for failure diagnosis in composite materials are well described. An extensive review of the use of NDT methods for material inspections was made by Gholizadeh [11]. Another review for non-destructive testing and evaluation (NDT\&E) methods applied in inspections of structural aircraft components was presented by Angelika [16]. In this review, applications of Visual and Penetrant Inspection, Tap-Testing, Eddy Current Inspection, Shearography, Thermography, Acoustic Emission Testing, and Ultrasonic Inspection were highlighted. More reviews on the use of NDT techniques for the composite materials, were also well documented [17-18]. Towsyfyan et al. [19] highlighted in sufficient details the success and challenges of different certified NDT methods that are applicable for damage detection and characterization in composite laminates used in the aircraft primary and secondary structures. Ehrhart et al. [20] summarized the NDT techniques that are, in particular, possibly viable for adhesively bonded composite materials. The summary focused on typical defects and quality management of composites, used in aerospace industry and substrates' applications, throughout the product life cycle.

\subsection{Applications of Thermography for Aircraft Composites}

Thermal and infrared testing (Infrared Thermography) is relatively considered as a newcomer to the world of NDT field. It is the process of monitoring the temperature variations of objects under test in the infrared portion of the electromagnetic spectrum; measuring and mapping thermal distributions from an object [21]. There are two types of thermographic methods for the inspection of the composites. The first type is the active method, where the thermal gradient is continuously produced/maintained using, for instance, cyclic stress. The second type is the passive method, which is the most widely used NDT technique in the area of composites inspection. Unlike the active method, the thermal gradient, in the passive method, results from transient changes. Ciampa et al. [22] discussed the very recent advances in active infrared thermography methods for aerospace components. Recent physical principle and thermal excitation sources in addition to traditional optically stimulated thermography, which uses external optical radiation such as flashes, heaters and laser systems, novel hybrid thermographic techniques were highlighted. Andrzej et al. [23] undertook an investigation for the damage Identification in Aircraft Composite Structures. Authors employed Piezoelectric Transducers (PZT) sensing, Ultrasonic, and Thermography to study the feasibility of these NDT methods for monitoring aircraft elements in varying environmental conditions. It was concluded that ultrasonic, and thermography showed an advantage over the PTZ method in detection and localization of introduced impact damage.

The detection of defects using the Infrared Thermography (IRT) was also investigated by Péronnet et al. [24]. In this investigation, three infrared thermographic techniques; IRT, Lock-in IRT and Pulse IRT, were applied to different types of composites that are used in aviation industry. Yet, tests involved both composites with small thicknesses and that one with large thicknesses. It was reported that Lock-in IRT method relatively showed higher accuracy comparing with the other methods. However, this was attributed to the optimization of the wave propagation, which eventually allows for a better crossing through the specimen under test. Montanini [25] also used lock-in and pulse phase infrared thermography to quantify subsurface defects in a reference specimen that was made out of Plexiglas. Thermal images recorded at different frequencies (frequency domain) were post-processed and direct measurement of material's thermal diffusivity was undertaken. Montanini postulated that the obtained results were very promising and offered the 
active thermography as a viable fast and powerful contactless NDT\&E method for the determination of subsurface defects.

Comparative results investigation between Infrared Thermography (IRT) and Electronic Speckle Pattern Interferometry (ESPI) for the NDT\&E of Aircraft composites was undertaken by Findeis et.al [26]. Throughout the experimental investigation using the proposed techniques, similar inspection methods were applied to the all composites under test. Findeis and his group reported that the proposed NDT techniques suite well the inspections of aircraft composites as they were able to locate the presence of all defects at different range of thermal load conditions, though the ESPI method showed relatively higher sensitivity than the classical IRT. Castanedo et al. [27] carried out another comparative study for the inspection of aerospace materials using pulsed thermography, lock-in thermography and vibrothermography. The proposed techniques were employed to inspect two typical aerospace parts; honeycomb structures and Glare. For pulsed and lock-in thermography, optical devices were used as a source of energy while mechanical waves were directly applied to the samples for vibrothermography method to stimulate the defects without heating the surfaces. Pulsed thermography method was also employed by Bendada et al. [28] for the evaluation of aircraft composites. Analytical solution using 3D thermal quadrupoles and mathematical perturbations was proposed to evaluate the depth and the volume of the flaw in a three-dimensional heat transfer configuration. To implement the proposed mathematical method, Laplace transform on the time variable $t$, is first applied and then a Fourier transform on the space variables, $x$ and $y$ is used. It should be noted that the volume of the flaw is defined as the flaw lateral extent times its thickness. This numerical method was used to solve data from anisotropic material containing a limited extent discontinuity. Authors postulated that the obtained theoretical results were then validated using an artificial sample that was previously inspected using infrared thermography test.

Hung et al. [29] presented a review and comparison investigation between shearography and active thermography for non-destructive and evaluation applications. Advantages, limitations and practical applications of the two techniques in the area of non-destructive evaluation of materials were well discussed. This involved the use of both techniques in aircraft industry. For instance, Hung pointed out that shearography technique was endorsed by the US-Federal Aviation Administration (FAA) as a compulsory NDT and evaluation method for inspecting, in particular, delamination's and other imperfections in aircraft tires. Also, Hung mentioned that active thermography method has been successfully employed for flaws inspection in many industrial applications, including aircraft industry. Acquired data from thermography tests are very often modulated due to the high contribution of the noise sources. External reflections, variations in the optical properties of the specimen and non-uniform heating are considered as main contributory noise sources. However, several signal processing methods were developed to handle this issue, and hence capability of detection and quantifying different damages could eventually be enhanced [30-31]. Vavilov et al. [32] provided a thorough review for the signal processing techniques widely employed for IRT data analysis. Feasibility of Gapped Smoothing Algorithm (GSA) for damage detection was investigated by Li et al. [33]. GSA method was applied to IRF data from aluminium pate and glass fibre with different defects such as flat bottom holes, material impurities and delamination. To generate the required heat, four heating lamps $(240 \mathrm{~V}, 275 \mathrm{~W})$ were employed and TIM-160 IR camera was used to collect the data. It was concluded that GSA was reliable and effective tool for identification subsurface damages.

For the detection of disbonds in an insulation, made out of low-density rubber, in solid rocket motors, Guo et al. [34] employed Pulsed Thermography (PT) method. Comparative results investigation between several data processing techniques was made. Authors reported that Signalto-Noise Ratio (SNR) will reach the highest value if a reference point in a defect free are of the specimen under test is predefined. Lopez et al. [35] optimized the processing of PT data acquired from composite fibre reinforced polymers (CFRP), with several simulated defects, using the Partial Least Square Thermography (PLST) method. It is worth to mention that PT data was collected by a FPA SBF125 IR camera and two photographic flash lamps were used to generate the heat. Lopez and his group claimed that the processed images with PLST method could result in an increase for $96 \%$ in the SNR. To quantify the material damages in carbon fibre reinforced polymers (CFRP) under 
impact, Chang el al. [36] used the Multi-Dimensional Ensemble Empirical Decomposition (MEEMD) algorithm. Also for CFRP subjected to impact test, the Coefficient Clustering Analysis (CSA) method integrated with PT was applied [37]. Initiation and propagation of fatigues were monitored using Pulsed Phase Thermography (PPT) method. A threshold was predefined to filter out immature failures [38]. Lock-In Thermography (LIT) was integrated with Acoustic Emission measurements to evaluate the fatigue limit on aircraft composite samples (on dog-bone aluminium and cross-ply $\mathrm{SiC} / \mathrm{BMAS}$ [ [39]. Grammatikos et al. [40] undertook an analysis for the durability and efficiency of the patch repair for an aluminium wing structure with CFRP composite patches using LIT method.

For better image contrast, and accordingly improved damage identification, Thermographic Signal Reconstruction (TSR) was applied to Step Heating (SHT) and Long Pulse Thermography (LPT) for damage inspections in aluminium and composite samples with flat bottom holes of different dimensions and depth [41]. Arora et al. [42] employed Frequency Modulated Thermography (FMT) method (time domain and frequency domain representations) to analyse an acquired data from CFRP samples with flat bottom holes. It was reported that the use of frequency domain (phase) method led to improvement in resolution and high sensitivity for damage detection. Sub-surface artificial defects in composite sandwich panels (commonly used materials in aircraft applications) could be identified using Laser-Line Thermography (LLT) [43-44]. Feasibility of Laser-Spot Thermography (LST), LaserLine Thermography (LLT) and Ultrasonic Stimulated Thermography (UST) for inspections of composite materials, employed in aircraft industry, such as metallic turbine blades, aluminium samples, CFRP composites etc., to name few, was also assessed in several investigations [45-49].

In deed several attempts were made to develop new methods to improve the overall performance of thermographic techniques. As well-known some of thermography methods employ external optical flashes to heat the surface of the specimen under test. The temperature decay curve is then recorded using IR camera. This method, in fact, allow for a good surface inspection only; deep damages may not easily be detected. In another words, the use of external optical flashes as heat sources leads to the detection of defects that are located just few millimetres from the sample surface. This can be attributed to the lack of a significant variation of the local temperatures nearby the subsurface cracks. To overcome this issue, an alternative method called Ultrasonic Stimulated Thermography (UST) is employed [50-52]. It should be telling that UST is also widely known as Thermosonics, Sonic IR or Vibro-Thermography. In UST method strong elastic vibration is generated within the sample test. Yet, this vibration will eventually cause very high frictional heat at the cracked surfaces that is measured using IR cameras with high temperature resolutions. One of the methods used to generate the vibration is to subject the surface under monitoring to an ultrasonic plastic welding horn. A high acoustic power (pulse power ranging from $1 \mathrm{~kW}$ to $80 \mathrm{~kW}$ ) with a frequency domain from $15 \mathrm{kHz}$ to $50 \mathrm{kHz}$ is applied over a predefined period of time (usually from $30-200$ milliseconds) [53]. Tremendous work to investigate the feasibility of UST method using the ultrasonic horn (as a mean of vibration excitation) for composite materials inspection was recently undertaken. A thermosonics inspection package integrated with a micro-bolometer array camera was developed by Polimeno et al. [52]. This compact inspection system was used to detect artificial delamination (embedded Teflon patch) in composites. Gaudenzi et al. [54] carried out a comparative study between the performance of UST, standard Optically Stimulated Thermography (OST) and ultrasonic phased array system for the inspection of CFRP samples that experienced low velocity impact damages. Findings of this study showed that material micro-defects could be quickly and accurately estimated using the UST method.

However, the use of ultrasonic horn to excite the vibration within the samples under test was reported as a causal factor for what-so-called acoustic chaos as a result of the coupling between material surface and the horn [52]. Yet, this phenomenon (acoustic chaos) will generate uncontrollable frequency components that may eventually lead the UST method to be incapable of (non-reproducible) detecting cracks if sufficient vibrations are not provided at the crack locations. Thus, a Local Damage Resonance (LDR) method has been proposed as an alternative mean for the material elastic effect instead of the ultrasonic horn. The use of LDR is basically intended to enhance the vibration amplitude at the damage locations only. This can be implemented as a result of the interaction between the acoustic/ultrasonic waves and the damaged area at frequencies that match 
the defect resonance [55-59]. Solodov et al. [60] employed Nonlinear Ultrasonic Stimulated Thermography (NUST) to improve the method for the selection of the driving frequency, which is used for the heat generation on the surface of materials undergone the test. It is worth to note that commercial loudspeakers were used to generate the required frictional heat. Authors postulated that the NUST results were similar to the results obtained by a shearography package. NUST was nonlinear narrow sweep excitation method by Fierro et al. [61] to detect debonds on aerospace composite stiffener panels and delamination fatigue damage on aluminium sample.

To stimulate the thermography measurements, Eddy Current Stimulated Thermography (ECST) method was used. Eddy currents are first introduced into the tested materials using coils and IR cameras are then employed to measure the associated induction heat; eddy current pulse with a frequency ranging from $150 \mathrm{kHz}$ to $450 \mathrm{kHz}$ over a short period of milliseconds is typically applied for the excitation [62]. In some research investigations, the ECST was integrated with the Principal Components Thermography (PCT) to analyse acquired data from CFRP samples impacted at different energies ranging from $4 \mathrm{~J}$ to 12J [63-64]. Analysis of phase images along with frequency domain techniques were employed to analyse ECST datasets acquired from delamination in CFRP samples [65-66]. Observations showed that the analysis of phase images, in particular, could periodically eliminate the influence of non-uniform heating, thus enhancing the detectability of the delamination in composites. Thomas et al. [67] designed a scanning induction method for ECST to inspect impact damages within CFRP samples. The method involved a continuously moving coil over the samples under test at an approximately speed of $40 \mathrm{~mm} / \mathrm{s}$. The resulting temperature profiles were then recorded using IR cameras. Yang et al. [68] reported that the combination of heating thermography through electromagnetic induction with cross correlation match filtering could alleviate not only the non-uniform heating but also lateral blurring effects that were typically noted when ECST method was used for damage inspections in CFRP.

The selection of the thermal excitation sources has to be give a para amount of importance. The reason behind this is that for the selection of proper thermal stimulation sources, factors such as clearance and accessibility of the samples, available power consumption, inspection cost, limitations, etc., to name a few, are considered. This has represented one interesting area of further research until today's date, and hence a number of studies have been undertaken to investigate the potential for improving/developing upon the existing thermal excitation methods. For instance, to directly tackle and respond to the lack of this engineering improvement, some studies have shedded light on the use of Microwave Thermography (MWT) and Material-Based Thermography approaches. To generate the required heat, the former basically employs electromagnetic radiation microwaves with high frequencies ranging from $300 \mathrm{MHz}$ to $30 \mathrm{GHz}$ [69]. The latter is based on either exploiting some specific properties of samples under examination and accordingly assess the internal health integrity; this method is known as a Direct Material-Based Thermography (DMT), or adding some thermosresistive components to the composites at the manufacturing stage, which will work as an internal thermal stimulation when thermography tests are performed; this approach is known as an Indirect Material-Based Thermography (DMT) [70]. Yang et al. [71] investigated the feasibility of MWT approach NDT applications and inspection of structural health integrity. It is worth mentioning that the application of MWT method in aerospace industry has only been introduced in the recent years. Comparative study between the MWT and CO2 Laser Infrared Thermography (LIT) for the analysis of defect detection due to impact in CFRP was done by Palumbo et al. [72]. Both approaches were reported as reliable tools for the detection of impact damages within CFRP though MWT needed less time for heating the samples (only two seconds) and simple signal processing analysis.

Sakagami et al. [73] performed an experimental work using two different Direct Material-Based Thermography (DMT) approaches. The first approach is called Singular Method and it is basically dependent on the increase of electrical current density near the tip of cracked regions to generate the required heat on electrically conductive materials under examination. The associated generated heat (concentration) can be easily detected from the top samples surface using IR camera. This method was applied to intralaminar crack tip at different angles of $0 \mathrm{o}, 35 \mathrm{o}, 45 \mathrm{o}, 60 \mathrm{o}$ and $90 \mathrm{o}$ in a stainless steel plate. It was concluded that this method led to less significant heat concentration when small inclination angles of crack were investigated, thus resulting in poor detection of cracks. Also was 
noted that $\sim 20 \mathrm{~A}$ input current is required for the singular method to detect the presence of the internal cracks. The second investigation involved the use of what-so-called Insulation Method. The authors developed this technique mainly for the detection of interlaminar cracks in CFRP composites. In this method, defects will act as an insulation as no singularity is generated within the temperature field. This will produce low temperature regions that can be detected from the top samples surface. Sakagami et al. employed this technique to detect a patch of polyimide film in a CFRP sample $(500 \mathrm{~mm}$ x 100mm) with 12 layers. Results revealed that this DMT method (insulation method) offers good alternative method for the inspection of internal flaws though high input current of $\sim 40 \mathrm{~A}$ must be supplied for high detection accuracy.

Internal health integrity of carbon fibres in CFRP composites has also been investigated using what-so-called Electrical Resistance Change Method (ERCM) and thermography [74-76]. The idea behind ERCM is to exploit the electrical conductivity of carbon fibres, which are used as sensors, and monitor any changes in the electrical resistance for cracks/damages identifications. ERCM has been integrated with thermography by Suzuki et al. [77-78] for the inspections of defects in two outermost layers of a composite laminate, aerospace composites, that were subjected to an electrical voltage. Suzuki et al. has recorded the varying temperature fields using IR camera. Another combination of thermography and ERCM was made by Grammatikos et al. [79] to undertake a comparative investigation between the available optical thermography techniques for aero-structures applications. Feasibility of techniques such as Pulsed Thermography (PT), Pulsed Phase Thermography (PPT) and Lock-In Thermograph (LIT) to determine the integrity of aircraft structure was investigated. In this work, a square electric pulse at low frequency was employed as a heat stimulation. Ahmed et al. [80] has also applied the metal based thermography for the inspection of composites using Heat Emitting Layer (HEL) technique. HEL employs additional layers embedded into laminate structure for rapid inspection of big regions. Orlowska et al. [81] has embedded a specifically designed 3D electrical grid made out of metal elements to produce low thermal density at damage regions. Authors used a long wave IR camera to acquire the data from the experimental tests.

As it can be seen that the literature, indeed, abounds with numerous attempts made not only to improve the existing thermography methods for the evaluation of aircraft composites but also to develop new techniques. However, these attempts were not only limited to the improve and/or develop new inspection tools rather they show that there are as well some studies have applied some well-established techniques for structural health monitoring. For instance, Avdelidis et al. [82-83] assessed the health status of aircraft composites using transient thermal NDT. Failure modes such as through skin sensing assessment of CFRP, impact damage on carbon fibre, drilling induced defects on multi-ply laminates of HEXCEL AS4/8552 carbon fibre composites, notches under multi-ply composite patching and simulated delamination between two plies on a multiply composite repair were investigated. Real time monitoring using pulsed thermography method was undertaken to record all emitting features from samples under examination. Authors claimed that the proposed thermal NDT approaches used in this research work are very promising in the area of inspection of various defects and/or subsurface features on aerospace composites.

In a research project, Kostopoulos et al. [84] employed a specifically designed robotic platform for autonomous monitoring of aeronautical composites. A combination of Infrared Thermography (IRT) and Phased Array (PA) was integrated with laser repair capabilities in this robotic scanner. It is worth to mention that this project is still on-going and aims to provide a reliable, rapid and robust tool for detection and characterization of different damages within aircraft materials. Deane et al. [85] also employed Unmanned Aerial Vehicle (UAV), integrated with a thermographic imaging system for the real-time inspection of aircraft CFRP panels. The investigation, in particular, focused on the use of pulsed thermography and vibrothermography. It was concluded that the UAV inspection approach could increase the probability of damage detection and significantly reduce the cost, time and workload of NDT inspections.

Machine learning algorithms were also employed to improve the feasibility of IRT for the inspection of composites. Example of this was the research work undertaken by Saeed et al. [86]. Artificial Neural Network (NN) was integrated with a line-scan thermography to detect different 
defects depth. This configuration was applied to specifically designed CFRP coupons with embedded and flat bottom holes' defects. It should be telling that a 3D printing method was used by the authors to manufacture test CFRP. Prior to the experimental analysis using the proposed NN method, a Multiphysics FEM simulation model of the inspection process was run to generate some training datasets for the assessment of the developed NN structure in a deterministic (noise free) environment. It was concluded that the detection accuracy of the proposed $\mathrm{NN}$ for the simulated data and the experimental data was $97 \%$ and $90 \%$ respectively. The artificial neural network algorithm was also combined with what so called Pulsed Thermal Ellipsometry (PTE) to investigate the fiber orientation on laminates reinforced with randomly-oriented strands [87]. Different CFRP samples were experimented using two thermography heating approaches. The first investigative step involved the use of traditional point heating source based on the Laser Spot Thermography Technique (LST) whilst the second method, proposed by the authors, is the line heating technique. This approach is basically based on the Laser Line Thermography Technique (LLT) and incorporated with an Artificial Neural Network (ANN) to ease the inspection of large areas in very short time. To properly heat the sample over a line region, Flying Laser Spot (FLS) method was used. The acquired thermal sequence was analyse using different image processing techniques such as Principal Component Thermography (PCT), Dynamic Thermal Tomography (DTT), Pulsed Phase Thermography (PPT). Authors reported that PCT showed an advantage over the other techniques as it recorded the highest accuracy of inspection in the training stage $(91.3 \%)$ and in the testing stage (71.6\%). Another multilayer Neural Network (NN) algorithm was selected to online predict different defect depths on carbon fiber samples [88]. The NN was linked line scan thermography setup to continuously transverse a controlled heater in tandem with a thermal detector in a linear $x-y-z$ stage. To implement the proposed inspection method datasets were simulated using a multiphysics. This simulated data was employed to train the proposed NN model which eventually was validated using the analysis of experimental datasets.

\subsection{Ultrasonic Testing (UT) and Acoustic Emission Technology (AE)}

Off the shelf, some research work has shown that the ultrasonic inspection technique could accurately detect a flaw in size, shape and depth for different kinds of composite structures used in the aeronautic industry [89-90]. Ultrasonic technique was discovered before the world second war and officially introduced by Japan shortly after to the international medical community. Ultrasonic testing (UT) uses very short range high frequency (frequency range lies between $1 \mathrm{MHz}$ to $25 \mathrm{MHz}$ or even above) ultrasonic waves [21]. Composite materials under NDT inspections will emit elastic waves (Lamb waves), which due to the anisotropic nature propagate in selective directions. Within the material under ultrasonic test, the elastic waves (Lamb waves) may produce three detection modes (reflection, transmission and backscattering) [91]. The feasibility of Lamb waves for the detection of different damage modes was discussed/summarised by Rose [92]. Su et al. [93] thoroughly summarized the generation and collection of UT data (Lamb wave-based damage identification approaches), selection of different modes, undertaking of modelling and simulation and eventually how the collected data is processed (using different signal processing techniques) and interpreted. In his PhD research work, Wronkowicz [94] utilized ultrasonic testing and image analysis as non-destructive evaluation tools for composite aircraft elements. The study concluded that accuracy, reliability and feasibility of ultrasonic testing for composite aircraft elements can be further improved with the use of image processing analysis.

Relatively similar to ultrasonic, Acoustic Emission (AE) is a phenomena of stress wave's generation due to a rapid release of energy from localized sources within a stressed material [95]. AE is considered as a real-time non-destructive evaluation (NDE) tool that can be used to detect $\mathrm{AE}$ events related to structural damages. If acoustic sensors are integrated with an effective signal processing system, AE can offer the user an ideal technique for real-time structural health monitoring [95]. For the aerospace applications, $\mathrm{AE}$ has been employed since several decades to monitor, for instance, airframe components in Air Force C-5A and Aircraft Transport C-135 [96]. Further, United 
States Air Force (USAF) has considered AE for monitoring F-105 fleet [96]. The feasibility of AE for modern monitoring of high performance aircrafts is still the subject of today's investigations. New published research work for instance includes the attempt by Tian et al. [97] where the Acoustic Emission (AE) technology was employed for the inspection of aircraft structures. AE beamforming method was proposed, in particular, to locate the AE sources emitting from damaged structures. Beamforming method based estimation can easily identify the location of sound signals from three dimensions near the monitored field. The obtained results were validated using several pencil lead break tests at various regions of a thin steel plate $(1000 \mathrm{~mm} \times 800 \mathrm{~mm})$. It is worth noting here that $\mathrm{AE}$ data was acquired at a sample rate of $3 \mathrm{MHz}$. Authors reached a conclusion that AE beamforming method could successfully identify locations of faulty structures. Also, the research work of Wang et al. [98] has focused on the use of piezoelectric (PZT) sensor network with shared signal transmission wires for active and passive monitoring of the health integrity of aircraft smart skin. The proposed method is basically dependent on the sharing of the transmitted signals between PZT sensors in a given network using signal transmission wires. A matrix of $(7 \times 7)$ PZT sensors, connected to each other via shared signal transmission wires, was designed and manufactured using Flexible Printed Circuit (FPC) process. It was reported that the use of FPC could lead to significant reduction in the weight of the manufactured layer. The authors postulated that the proposed method could identify the actual locations of impacts and/or damages on a glass fiber reinforced plate with an effective monitoring region of $(780 \mathrm{~mm} \times 780 \mathrm{~mm})$. The topic estimation of remaining useful life for aircraft structures was also the subject of intensive research. Well planned and timely prognosis of aircrafts components will ensure safe flights, scheduled maintenance activities and low downtime. Ul-Hassan et al. [99] undertook a comparative investigation between different programs that are possibly viable for the prediction of crack propagation in aircraft wings. The proposed framework was applied to flaw data acquired from countersunk rivet-holes of an in-service Airbus A310 aircraft's wing.

\subsection{Eddy Current Testing (ECT)}

Eddy Current Testing (ECT) employs electromagnetic induction to detect defects in an electrically conductive material. In ECT, when a conductive material carrying alternating current is bought in proximity to the test specimen, it generates eddy current and consequences of these eddy currents are measured. It can also be described as the use of the effect of electromagnetic fields and induction to characterize physical properties of metallic materials [21, 100]. An impedance plane plot is very often used to represent the ECT results. The coil resistance and inductive reactance are plotted on x-axis and y-respectively. And the shape of the resulting ECT impedance graph similar to Lissajous figures; generally, visual observations from this graph are used to interpret and spot the defects [101]. A Pulsed Eddy Current (PEC) sensor was employed in a specifically designed test-rig by Sophian et al. [102] for the detection of lap-joint structures in aircrafts. One of the reported advantages of this sensor is the use of new excitation circuit that does not need any additional signal amplification. Statistical features such as peak value in both time and frequency representations were extracted from the acquired data. Initial observations from this study showed that the proposed current system could detect surface and subsurface cracks. Further signal processing analysis could also ease the identification of defect locations and alleviate the noise in the modulated data.

To improve the processing of measured data obtained from NDT's in aerospace applications, D'Angelo et al. [103] applied mathematical Genetic Programming (GP) model to a publicly available Eddy-Current dataset. Evaluation/assessment of the obtained results was undertaken using the wellestablished metrics for assessing binary classifiers. The authors stated that the proposed GP classifiers have showed an advantage over the other known approaches when they are compared against the inaccuracy, uncertainty and the presence of noise in data. Furthermore, the proposed GP-based Classifier (GPC) could successfully extract the complex insights (information) from the selected NDT features more efficiently. It should be telling that the results were represented on the impedance plane plot and the proposed analysis approach presented in [101, 104-106], which is highly depending on the characterization of Lissajous shapes using some geometric features, was employed to avoid the similarity among Lissajous shapes, the presence of noise and high number of measurement points 
that may belong to either different defects or to the presence of noise; thus leading to inaccurate visual interpretation. The ECT has also been employed to detect gaps between CFRP composites as a result of overlapping of assembly parts with geometrical changes [107]. Two overlapped reinforced plastic (CFRP) tapes were tested and the thickness of the gaps was changed by increasing the number of PVS sheets between the tapes. The extracted ECT features were used to train some machine learning classifiers, which have accordingly been used to differentiate the gaps and the obtained results were validated through the 10-fold cross validation method. Li et al. [108] quantitatively evaluated damages in CFRP plates, also widely known as green aircraft production, using a non-contact electromagnetic (EM) sensor with coupled spiral inductors (CSI). Under induced impact and simulated damages, locations, depth and width of subsurface defects were determined. Further, signal features such as inspection frequency, standoff distance and signal power were also investigated prior testing. Authors reported that analysis of signal features has eased the experimental setup and the sensor calibration.

The lack of an appropriate adhesive bonding for load critical CFRP primary structures, which may eventually cause a surface contamination, led De Vito et al. [109-110] to propose an electronic nose structure, a hybrid Airsense GDA2 electronic nose sensor array, as NDT tool for the inspection of pre-bond surface. The GDA2 configuration consisted of two Metal Oxide (MOX) sensors, one Electrochemical (EC) sensor, one Photo Ionization Detector (PID) sensor and an integrated Ion Mobility Spectrometer (IMS) sensor. This platform was integrated with an ad-hoc pattern recognition system, combination of real time classifiers and implementing a reject option, to rapidly determine the contamination conditions. This configuration was used to investigate three different contamination levels. It is worth to mention that moisture, hydraulic fluids and release agents were employed to simulate the contamination scenarios. Findings of this study showed that the proposed method could achieve $78 \%$ of classification accuracy with $14 \%$ false positive rate and $0 \%$ false negative rate. Dilonardo et al. [111] proposed X-ray Computed Tomography (CT) as a versatile NDT inspection technique for health integrity of aircraft CFRP composites. The method was applied to assess the status of porosity levels in two different aircraft CFRP materials (layered composite and sandwich structure). The investigation included the study of volume distribution of the individuated pores/voids that allowed for interactive 3D exploration and quantitative analysis for the porosity levels. The authors postulated that the proposed CT technique along with the selected data analysis method in this work could provide sufficient details about size, location and shape for failure modes such as pores and misalignment in CFRP under severe stress conditions; thus it could be considered as one of good alternative tools for the classical NDT inspection methods (authors' view).

\section{3. Conclusion}

There is a tremendous published work, off the shelf, in the literature that needs to be summarised in one document. This paper attempted to present an overview of the most published research work that investigated the feasibility of well-established NDT techniques and some other developed tools for the inspection and evaluation of aerospace components. The review also highlighted some signal processing method and machine learning algorithms which were combined with these NDT techniques to ease and increase their capabilities in the inspection of composite damages. With so many different techniques, each having their own characteristics, some of them might be possibly viable for certain applications but utterly infeasible in other cases. It was seen that some of these techniques were reported as a rapid, contactless, relatively low-cost compared to the others and, when they are integrated with efficient and advanced data processing tools for the inspection of composites' defects such as flaws at surface and subsurface of the metals, between surface coatings, delamination, debonding, corrosion and fatigue damage etc., to name a few, they could offer the end-users very reliable and robust and high accurate defect identification techniques. However, some developed/proposed methods are still in their infancy for the NDT\&E of aerospace composites. Hence, when preparing the inspection plan, it is very essential to be well advised/experienced of the selection of the appropriate NDT technique. This is very critical and must 
be given a para amount of importance; thus optimizing and ensuring a high accuracy level for the overall inspection outcomes.

Author Contributions: Conceptualization, M.A. and L.F.; methodology, M.A.; validation, L.F. and N.A.; investigation, L.F.; resources, M.A.; data curation, M.A.; writing-original draft preparation, M.A.; writingreview and editing, L.F.; visualization, N.A.; supervision, L.F. and N.A.; funding acquisition, M.A.

Funding: This research received no external funding.

Conflicts of Interest: Declare conflicts of interest or state.

\section{References}

1. Kapadia, A. Best Practice Guide, Non-Destructive Testing of Composite Materials. https://avaloncsl.files.wordpress.com/2013/01/ncn-best-practice-ndt.pdf, (accessed April 2020).

2. Chawla, K. K.; Springer Chemistry and Materials Science eBooks 2012 English/International., Composite materials science and engineering. 3rd ed.; Springer Science+Business Media,: New York, 2012.

3. Nicolais, L.; Nicolais, L.; Meo, M.; Milella, E.; SpringerLink, Composite Materials : A Vision for the Future. 1st ed.; 2011.

4. Baker, A. A.; Baker, A. A.; Dutton, S.; Kelly, D.; American Institute of, A.; Astronautics, Composite materials for aircraft structures. 2004.

5. Kar, K. K.; SpringerLink (Online service), Composite Materials Processing, Applications, Characterizations. Springer Berlin Heidelberg : Imprint: Springer,: Berlin, Heidelberg, 2017; pp. XVII, 686 p. 382 illus., 135 illus. in color. online resource.

6. Ajay Kumar, P.; Rohatgi, P.; Weiss, D., 50 Years of Foundry-Produced Metal Matrix Composites and Future Opportunities. International Journal of Metalcasting 2020, 14 (2), 291-317.

7. Campbell, F. C., Manufacturing Technology for Aerospace Structural Materials. Elsevier Science: 2006.

8. Statista Distribution of composite materials used in commercial aircraft manufacturing worldwide from 1985 to 2014. https://www.statista.com/statistics/954913/share-composites-in-aircraftdesign/\#statisticContainer (accessed April 2020).

9. Makhlouf, A. S. H.; Aliofkhazraei, M., Handbook of Materials Failure Analysis with Case Studies from the Construction Industries. Vol. 6. Butterworth-Heinemann Imprint Elsevier Science \& Technology Books, Elsevier Science \& Technology Distributor: San Diego, Oxford, 2018; p. 560 p.

10. Wang, B.; Zhong, S.; Lee, T.-L.; Fancey, K. S.; Mi, J., Non-destructive testing and evaluation of composite materials/structures: A state-of-the-art review. Advances in Mechanical Engineering 2020, 12 (4), 1687814020913761.

11. Prof Prakash, R., Non Destructive Testing Techniques. New Academic Science: Kent, 2009; p. 140 p. 111.

12. Gholizadeh, S., A review of non-destructive testing methods of composite materials. Procedia Structural Integrity 2016, 1, 50 - 57.

13. Inspectioneering Overview $\quad$ Non-destructive $\quad$ Testing https://inspectioneering.com/tag/nondestructive+testing (accessed April 2020).

14. NUCLEOM Types of Non-Destructive Testing. https://nucleom.ca/en/knowledge/types-of-nondestructive-testing/ (accessed April 2020).

15. Dubov, A.; Vlasov, V., About the New Classification of NDT Methods Based on Positions of Risks and Equipment Life Assessment. In 5th International Conference on Certification and Standardization in NDT, Berlin, 2007.

16. Wronkowicz-Katunin, A., A Brief Review on NDT\&E Methods For Structural Aircraft Components. Fatigue of Aircraft Structures 2018, 2018 (10), 73.

17. Akademia Baru, P.; Khattak, M.; Mukhtar, A.; Shahid, I.; Sufian, S. M., A Review on Application of Non Destructive Techniques on Composites. 2016, 12-21.

18. Vavilov, V. P.; Burleigh, D. D., Review of pulsed thermal NDT: Physical principles, theory and data processing. NDT \& E International 2015, 73, 28-52.

19. Towsyfyan, H.; Biguri, A.; Boardman, R.; Blumensath, T., Successes and challenges in non-destructive testing of aircraft composite structures. Chinese Journal of Aeronautics 2020, 33 (3), 771-791.

20. Ehrhart, B.; Valeske, B.; Bockenheimer, C., 9 - Non-destructive evaluation (NDE) of aerospace composites: methods for testing adhesively bonded composites. In Non-Destructive Evaluation (NDE) of Polymer Matrix Composites, Karbhari, V. M., Ed. Woodhead Publishing: 2013; pp 220-237. 
21. BINDT, Non-Destructive Testing (NDT) - Guidance Document: An Introduction to NDT Common Methods. The British Institute of Non-Destructive Testing: November 2015; Vol. 2.

22. Ciampa, F.; Mahmoodi, P.; Pinto, F.; Meo, M., Recent Advances in Active Infrared Thermography for NonDestructive Testing of Aerospace Components. Sensors 2018, 18 (2), 609.

23. Katunin, A.; Dragan, K.; Dziendzikowski, M., Damage identification in aircraft composite structures: A case study using various non-destructive testing techniques. Composite Structures 2015, 127, 1-9.

24. Péronnet, E.; Pastor, M.; Huillery, R.; Dalverny, O.; Mistou, S.; Welemane, H., Non-Destructive Investigation of Defects in Composite Structures by Three Infrared Thermographic Techniques. In 15th International Conference on Experimental Mechanics, Porto, Portugal 2012.

25. Montanini, R., Quantitative determination of subsurface defects in a reference specimen made of Plexiglas by means of lock-in and pulse phase infrared thermography. Infrared Physics \& Technology 2010, 53 (5), 363-371.

26. Findeis, D.; Gryzagoridis, J.; Lombe, C., Comparing Infrared Thermography and ESPI for NDE of Aircraft Composites. Insight - Non-Destructive Testing and Condition Monitoring 2010, 52 (5).

27. Ibarra-Castanedo, C.; Genest, M.; Guibert, S.; Piau, J.-M.; Maldague, X.; Bendada, A., Inspection of aerospace materials by pulsed thermography, lock-in thermography, and vibrothermography: a comparative study. SPIE: 2007; Vol. 6541.

28. Bendada, A.; Erchiqui, F.; Lamontagne, M., Pulsed thermography in the evaluation of an aircraft composite using 3D thermal quadrupoles and mathematical perturbations. Inverse Problems 2005, 21 (3), 857-877.

29. Chen, Y.; Hung, Y.; Ng, S.; Huang, Y.; Liu, L., Review and comparison of shearography and active thermography for nondestructive testing and evaluation (NDT\&E). SPIE: 2009; Vol. 7375.

30. Zalameda, J.; Rajic, N.; Winfree, W., A comparison of image processing algorithms for thermal nondestructive evaluation. SPIE: 2003; Vol. 5073.

31. Madruga, F. J.; Ibarra-Castanedo, C.; Conde, O. M.; López-Higuera, J. M.; Maldague, X., Infrared thermography processing based on higher-order statistics. NDT \& E International 2010, 43 (8), 661-666.

32. Vavilov, V. P.; Burleigh, D. D., Review of pulsed thermal NDT: Physical principles, theory and data processing. NDT \& E International 2015, 73, 28 - 52.

33. Li, B.; Ye, L.; Li, E.; Shou, D.; Li, Z.; Chang, L., Gapped smoothing algorithm applied to defect identification using pulsed thermography. Nondestructive Testing and Evaluation 2015, 30 (2), 171-195.

34. Guo, X.; Vavilov, V., Pulsed thermographic evaluation of disbonds in the insulation of solid rocket motors made of elastomers. Polymer Testing 2015, 45, 31-40.

35. Lopez, F.; Ibarra-Castanedo, C.; de Paulo Nicolau, V.; Maldague, X., Optimization of pulsed thermography inspection by partial least-squares regression. NDT \& E International 2014, 66, 128-138.

36. Chang, Y.-S.; Yan, Z.; Wang, K.-H.; Yao, Y., Non-destructive testing of CFRP using pulsed thermography and multi-dimensional ensemble empirical mode decomposition. Journal of the Taiwan Institute of Chemical Engineers 2016, 61, 54-63.

37. Zhao, Y.; Tinsley, L.; Addepalli, S.; Mehnen, J.; Roy, R., A coefficient clustering analysis for damage assessment of composites based on pulsed thermographic inspection. NDT \& E International 2016, 83, 5967.

38. Shin, P. H.; Webb, S. C.; Peters, K. J., Pulsed phase thermography imaging of fatigue-loaded composite adhesively bonded joints. NDT \& E International 2016, 79, 7-16.

39. Kordatos, E. Z.; Dassios, K. G.; Aggelis, D. G.; Matikas, T. E., Rapid evaluation of the fatigue limit in composites using infrared lock-in thermography and acoustic emission. Mechanics Research Communications 2013, 54, 14-20.

40. Grammatikos, S. A.; Kordatos, E. Z.; Matikas, T. E.; Paipetis, A. S., Service and maintenance damage assessment of composite structures using various modes of infrared thermography. IOP Conference Series: Materials Science and Engineering 2015, 74, 012006.

41. Almond, D. P.; Angioni, S. L.; Pickering, S. G., Long pulse excitation thermographic non-destructive evaluation. NDT \& E International 2017, 87, 7-14.

42. Arora, V.; Siddiqui, J. A.; Mulaveesala, R.; Muniyappa, A., Pulse Compression Approach to Nonstationary Infrared Thermal Wave Imaging for Nondestructive Testing of Carbon Fiber Reinforced Polymers. IEEE Sensors Journal 2015, 15 (2), 663-664.

43. Ley, O.; Butera, M.; Godinez, V., Inspection of composite structures using line scanning thermography. SPIE: 2012; Vol. 8354. 
44. Ley, O.; Godinez, V., Line Scanning Thermography and its Application Inspecting Aerospace Composites. In In Proceedings of the 5th International Symposium on NDT in Aerospace, Singapore, 2013.

45. Fernandes, H.; Zhang, H.; Figueiredo, A.; Malheiros, F.; Ignacio, L. H.; Sfarra, S.; IbarraCastanedo, C.; Guimaraes, G.; Maldague, X., Machine Learning and Infrared Thermography for Fiber Orientation Assessment on Randomly-Oriented Strands Parts. Sensors (Basel) 2018, 18 (1), 288.

46. Zhang, H.; Fernandes, H.; Djupkep Dizeu, F. B.; Hassler, U.; Fleuret, J.; Genest, M.; IbarraCastanedo, C.; Robitaille, F.; Joncas, S.; Maldague, X., Pulsed micro-laser line thermography on submillimeter porosity in carbon fiber reinforced polymer composites: experimental and numerical analyses for the capability of detection. Appl. Opt. 2016, 55 (34), D1-D10.

47. Khodayar, F.; Lopez, F.; Ibarra-Castanedo, C.; Maldague, X., Optimization of the Inspection of Large Composite Materials Using Robotized Line Scan Thermography. Journal of Nondestructive Evaluation 2017, 36 (2), 32.

48. Fedala, Y.; Streza, M.; Sepulveda, F.; Roger, J. P.; Tessier, G.; Boué, C., Infrared Lock-in Thermography Crack Localization on Metallic Surfaces for Industrial Diagnosis. Journal of Nondestructive Evaluation 2014, 33 (3), 335-341.

49. Roemer, J.; Uhl, T.; Pieczonka, L., Laser spot thermography for crack detection in aluminum structures. 2015.

50. Barden, T.; Almond, D.; Pickering, S.; Morbidini, M.; Cawley, P., Detection of impact damage in CFRP composites by thermosonics. Nondestructive Testing and Evaluation - Nondestruct Test Eval 2007, 22, 7182.

51. Sakagami, T.; Kuroki, K.; Kubo, S.; Katsumata, R.; Matsumoto, Y.; Harada, Y., Detection of Stress Corrosion Cracking by Sonic-IR Technique. In In Proceedings of the 7th International Conference on NDE in Relation to Structural Integrity for Nuclear and Pressurized Components, Yokohama, Japan, 2009.

52. Polimeno, U.; Almond, D. P.; Weekes, B.; Chen, E. W. J., A compact thermosonic inspection system for the inspection of composites. Composites Part B: Engineering 2014, 59, 67-73.

53. Umar, M. Z.; Vavilov, V.; Abdullah, H.; Ariffin, A. K., Ultrasonic infrared thermography in non-destructive testing: A review. Russian Journal of Nondestructive Testing 2016, 52 (4), 212-219.

54. Gaudenzi, P.; Bernabei, M.; Dati, E.; De Angelis, G.; Marrone, M.; Lampani, L., On the evaluation of impact damage on composite materials by comparing different NDI techniques. Composite Structures $2014,118,257-266$.

55. Rahammer, M.; Solodov, I.; Bisle, W.; Scherling, D.; Kreutzbruck, M., Thermosonic Testing with Phase Matched Guided Wave Excitation. Journal of Nondestructive Evaluation 2016, 35 (3), 47.

56. De Angelis, G.; Meo, M.; Almond, D. P.; Pickering, S. G.; Angioni, S. L., A new technique to detect defect size and depth in composite structures using digital shearography and unconstrained optimization. NDT \& E International 2012, 45 (1), 91-96.

57. Solodov, I.; Bai, J.; Busse, G., Resonant ultrasound spectroscopy of defects: Case study of flat-bottomed holes. Journal of Applied Physics 2013, 113 (22), 223512.

58. Ciampa, F.; Scarselli, G.; Meo, M., On the generation of nonlinear damage resonance intermodulation for elastic wave spectroscopy. J Acoust Soc Am 2017, 141 (4), 2364.

59. Zalameda, J.; Winfree, W.; Yost, W., Air coupled acoustic thermography (acat) inspection technique. AIP Conference Proceedings 2008, 975, 467-474.

60. Solodov, I.; Rahammer, M.; Gulnizkij, N.; Kreutzbruck, M., Noncontact Sonic NDE and Defect Imaging Via Local Defect Resonance. Journal of Nondestructive Evaluation 2016, 35 (3), 48.

61. Fierro, G. P. M.; Calla, D.; Ginzburg, D.; Ciampa, F.; Meo, M., Nonlinear ultrasonic stimulated thermography for damage assessment in isotropic fatigued structures. Journal of Sound and Vibration 2017, 404, 102-115.

62. Zainal Abidin, I.; Yun Tian, G.; Wilson, J.; Yang, S.; Almond, D., Quantitative evaluation of angular defects by pulsed eddy current thermography. NDT \& E International 2010, 43 (7), 537-546.

63. He, Y.; Tian, G.; Pan, M.; Chen, D., Impact evaluation in carbon fiber reinforced plastic (CFRP) laminates using eddy current pulsed thermography. Composite Structures 2014, 109, 1-7.

64. Ren, W.; Liu, J.; Tian, G. Y.; Gao, B.; Cheng, L.; Yang, H., Quantitative non-destructive evaluation method for impact damage using eddy current pulsed thermography. Composites Part B: Engineering 2013, 54, 169-179. 
65. He, Y.; Yang, R., Eddy Current Volume Heating Thermography and Phase Analysis for Imaging Characterization of Interface Delamination in CFRP. IEEE Transactions on Industrial Informatics 2015, 11 (6), 1287-1297.

66. Ishikawa, M.; Shiiya, Y.; Kogo, Y.; Hatta, H.; Habuka, Y.; Fukui, R.; Utsunomiya, S., Nondestructive inspection of CFRP laminates using phase-transformed induction heating thermography. Transactions of the JSME (in Japanese) 2015, 81.

67. Kidangan, R.; Balasubramaniam, K., Scanning induction thermography (SIT) for imaging damages in carbon-fibre reinforced plastics (CFRP) components. 2015; Vol. 1650.

68. Yang, R.; He, Y., Polymer-matrix composites carbon fibre characterisation and damage inspection using selectively heating thermography (SeHT) through electromagnetic induction. Composite Structures 2016, 140, 590-601.

69. Foudazi, A.; Donnell, K. M.; Ghasr, M. T. In Application of Active Microwave Thermography to delamination detection, 2014 IEEE International Instrumentation and Measurement Technology Conference (I2MTC) Proceedings, 12-15 May 2014; 2014; pp 1567-1571.

70. Yang, R.; He, Y., Optically and non-optically excited thermography for composites: A review. Infrared Physics \& Technology 2016, 75, 26-50.

71. Yang, R.; Zhang, H.; Li, T.; He, Y. In An investigation and review into microwave thermography for NDT and SHM, 2015 IEEE Far East NDT New Technology \& Application Forum (FENDT), 28-31 May 2015; 2015; pp 133-137.

72. Keo, S. A.; Defer, D.; Breaban, F.; Brachelet, F., Comparison between Microwave Infrared Thermography and CO2 Laser Infrared Thermography in Defect Detection in Applications with CFRP. Materials Sciences and Applications 2013, 4, 600-605.

73. Sakagami, T.; Ogura, K., A New Flaw Inspection Technique Based on Infrared Thermal Images Under Joule Effect Heating. Transactions of the Japan Society of Mechanical Engineers Series A 1992, 58 (555), 22242231.

74. Suzuki, Y.; Todoroki, A.; Mizutani, Y.; Matsuzaki, R., Impact Damage Detection in CFRP Using Statistical Analysis of Resistance-Temperature Characteristics. Journal of Solid Mechanics and Materials Engineering 2011, 5 (1), 33-43.

75. Abry, J. C.; Bochard, S.; Chateauminois, A.; Salvia, M.; Giraud, G., In situ detection of damage in CFRP laminates by electrical resistance measurements. Composites Science and Technology 1999, 59 (6), 925-935.

76. Schulte, K.; Baron, C., Load and failure analyses of CFRP laminates by means of electrical resistivity measurements. Composites Science and Technology 1989, 36 (1), 63-76.

77. Suzuki, Y.; Todoroki, A.; Matsuzaki, R.; Mizutani, Y., Impact-damage visualization in CFRP by resistive heating: Development of a new detection method for indentations caused by impact loads. Composites Part A: Applied Science and Manufacturing 2012, 43 (1), 53-64.

78. Suzuki, Y.; Todoroki, A.; Matsuzaki, R.; Mizutani, Y., Indentation-Damage Visualization in CFRP by Resistive Heating: Analytical Verification of The Inspection of Aircraft Using Its Lightning Protection Systems. Journal of Solid Mechanics and Materials Engineering 2012, 6 (3), 213-226.

79. Grammatikos, S. A.; Kordatos, E. Z.; Barkoula, N. M.; Matikas, T. E.; Paipetis, A. S., Innovative nondestructive evaluation and damage characterisation of composite aerostructures using thermography. Plastics, Rubber and Composites 2011, 40 (6-7), 342-348.

80. Ahmed, T. J.; Nino, G. F.; Bersee, H. E. N.; Beukers, A., Heat emitting layers for enhancing NDE of composite structures. Composites Part A: Applied Science and Manufacturing 2008, 39 (6), 1025-1036.

81. Orłowska, A.; Kołakowski, P.; Holnicki-Szulc, J., Detecting delamination zones in composites by embedded electrical grid and thermographic methods. Smart Materials and Structures 2011, 20 (10), 105009.

82. Avdelidis, N. P.; Almond, D. P.; Dobbinson, A.; Hawtin, B. C.; Ibarra-Castanedo, C.; Maldague, X., Aircraft composites assessment by means of transient thermal NDT. Progress in Aerospace Sciences 2004, 40 (3), 143-162.

83. Avdelidis, N. P.; Hawtin, B. C.; Almond, D. P., Transient thermography in the assessment of defects of aircraft composites. NDT \& E International 2003, 36 (6), 433-439.

84. Kostopoulos, V.; Psarras, S.; Loutas, T.; Sotiriadis, G.; Gray, I.; Padiyar, M. J.; Petrunin, I.; Raposo, J.; Fragonara, L. Z.; Tzitzilonis, V.; Dassios, K.; Exarchos, D.; Andrikopoulos, G.; Nikolakopoulos, G., Autonomous Inspection and Repair of Aircraft Composite Structures. IFACPapersOnLine 2018, 51 (30), 554-557. 
85. Deane, S.; Avdelidis, N. P.; Ibarra-Castanedo, C.; Zhang, H.; Yazdani Nezhad, H.; Williamson, A. A.; Mackley, T.; Davis, M. J.; Maldague, X.; Tsourdos, A., Application of NDT thermographic imaging of aerospace structures. Infrared Physics \& Technology 2019, 97, 456-466.

86. Saeed, N.; Abdulrahman, Y.; Amer, S.; Omar, M. A., Experimentally validated defect depth estimation using artificial neural network in pulsed thermography. Infrared Physics \& Technology 2019, 98, 192-200.

87. Fernandes, H.; Zhang, H.; Figueiredo, A.; Malheiros, F.; Ignacio, L. H.; Sfarra, S.; IbarraCastanedo, C.; Guimaraes, G.; Maldague, X., Machine Learning and Infrared Thermography for Fiber Orientation Assessment on Randomly-Oriented Strands Parts. Sensors (Basel) 2018, 18 (1).

88. Saeed, N.; Omar, M. A.; Abdulrahman, Y., A neural network approach for quantifying defects depth, for nondestructive testing thermograms. Infrared Physics \& Technology 2018, 94, 55-64.

89. Garnier, C.; Pastor, M.-L.; Eyma, F.; Lorrain, B., The detection of aeronautical defects in situ on composite structures using Non Destructive Testing. Composite Structures 2011, 93 (5), 1328-1336.

90. Djordjevic, B. Ultrasonic characterization of advanced composite materials. The 10th International Conference of the Slovenian Society for Non-Destructive Testing, Ljubljana, Slovenia 2009.

91. Wang, B.; Zhong, S.; Lee, T.; Fancey, K.; Mi, J., Non-destructive testing and evaluation of composite materials/structures: A state-of-the-art review. 2020; Vol. 12.

92. Rose, J. L., A Baseline and Vision of Ultrasonic Guided Wave Inspection Potential. Journal of Pressure Vessel Technology 2002, 124 (3), 273-282.

93. Su, Z.; Ye, L.; Lu, Y., Guided Lamb waves for identification of damage in composite structures: A review. Journal of Sound and Vibration 2006, 295 (3), 753-780.

94. Wronkowicz, A. Non-destructive Evaluation of Composite Aircraft Elements Based on Ultrasonic Testing and Image Analysis. Politechnika Śląska, Gliwice.

95. Elforjani, M.; Mba, D., Monitoring the Onset and Propagation of Natural Degradation Process in a Slow Speed Rolling Element Bearing With Acoustic Emission. Journal of Vibration and Acoustics 2008, 130 (4).

96. Chuang, S., Y., Review of Progress in Quantitative Nondestructive Evaluation, Chapter 1: General Techniques-Fundamentals, Section: Acoustic Emission. Springer-Verlag US: La Jolla, CA.

97. Tian, H.; Qiang, P.; Xiandong, L.; Yingchun, S., AE beamforming method for damage inspection of aircraft structures. Procedia Engineering 2011, 17, 297-302.

98. Wang, Y.; Qiu, L.; Luo, Y.; Ding, R.; Jiang, F., A piezoelectric sensor network with shared signal transmission wires for structural health monitoring of aircraft smart skin. Mechanical Systems and Signal Processing 2020, 141, 106730.

99. Hassan, M. u.; Danish, F.; Yousuf, W. B.; Khan, T. M. R., Comparison of different life distribution schemes for prediction of crack propagation in an aircraft wing. Engineering Failure Analysis 2019, 96, 241-254.

100. Bowler, N., Eddy-Current Nondestructive Evaluation. 1st ed.; Springer-Verlag New York: 2019. https://www.springer.com/gp/book/9781493996278.

101. Angelo, G. D.; Laracca, M.; Rampone, S. In Automated Eddy Current non-destructive testing through low definition lissajous figures, 2016 IEEE Metrology for Aerospace (MetroAeroSpace), 22-23 June 2016; 2016; pp 280-285.

102. Sophian, A.; Tian, G. Y.; Taylor, D.; Rudlin, J., Design of a pulsed eddy current sensor for detection of defects in aircraft lap-joints. Sensors and Actuators A: Physical 2002, 101 (1), 92-98.

103. D'Angelo, G.; Palmieri, F., Knowledge elicitation based on genetic programming for non destructive testing of critical aerospace systems. Future Generation Computer Systems 2020, 102, 633-642.

104. Angelo, G. D.; Rampone, S. In Diagnosis of aerospace structure defects by a HPC implemented soft computing algorithm, 2014 IEEE Metrology for Aerospace (MetroAeroSpace), 29-30 May 2014; 2014; pp 408-412.

105. Angelo, G. D.; Rampone, S. In Shape-based defect classification for non destructive testing, 2015 IEEE Metrology for Aerospace (MetroAeroSpace), 4-5 June 2015; 2015; pp 406-410.

106. D'Angelo, G.; Laracca, M.; Rampone, S.; Betta, G., Fast Eddy Current Testing Defect Classification Using Lissajous Figures. IEEE Transactions on Instrumentation and Measurement 2018, 67 (4), 821-830.

107. Angelo, G. D.; Cavaccini, G.; Rampone, S. In Shimming Analysis of Carbon-Fiber Composite Materials with Eddy Current Testing, 2018 5th IEEE International Workshop on Metrology for AeroSpace (MetroAeroSpace), 20-22 June 2018; 2018; pp 68-73.

108. Li, Z.; Haigh, A.; Soutis, C.; Gibson, A.; Sloan, R.; Karimian, N., Detection and evaluation of damage in aircraft composites using electromagnetically coupled inductors. Composite Structures 2016, 140, 252 261. 
109. De Vito, S.; Massera, E.; Miglietta, M.; Di Palma, P.; Fattoruso, G.; Brune, K.; Di Francia, G., Detection and quantification of composite surface contaminants with an e-nose for fast and reliable prebond quality assessment of aircraft components. Sensors and Actuators B: Chemical 2016, 222, 1264-1273.

110. De Vito, S.; Massera, E.; Miglietta, M.; Fattoruso, G.; Di Francia, G., Electronic Nose as an NDT Tool for Aerospace Industry. Physics Procedia 2015, 62, 23-28.

111. Dilonardo, E.; Nacucchi, M.; De Pascalis, F.; Zarrelli, M.; Giannini, C., High resolution X-ray computed tomography: A versatile non-destructive tool to characterize CFRP-based aircraft composite elements. Composites Science and Technology 2020, 192, 108093. 